\title{
Unilateral twin ectopic pregnancy managed with laparoscopic salpingectomy
}

\author{
M. Raja • B. Donepudi • B. Whitlow
}

Received: 11 March 2007 / Accepted: 14 June 2007 /Published online: 4 August 2007

(C) Springer-Verlag 2007

\begin{abstract}
We describe a rare case of unilateral twin tubal ectopic pregnancy in a multiparous lady in the right fallopian tube that was confirmed on laparoscopy and managed by laparoscopic salpingectomy.
\end{abstract}

Keywords Twin ectopic pregnancy · Laparoscopy ·

Salpingectomy

\section{Introduction}

Ectopic pregnancy can pose a diagnostic and therapeutic challenge to a clinician as the presenting symptoms and signs vary widely between patients and also because of the potential mortality and morbidity associated with the condition. The relative risk of death is 10 times greater than that for childbirth and about 50 times greater than that for an induced abortion. With the advances in technology and the availability of high-resolution TV probes it is increasingly possible to diagnose the ectopic pregnancies at an earlier stage and hence the classic triad of early pregnancy uterine bleeding, pelvic pain and adnexal mass is not commonly seen. Although the incidence of ectopic pregnancy is increasing and is known to be around $0.5-1.0 \%$ of all pregnancies, the chances of a spontaneous twin tubal ectopic pregnancy is only 1:20,000 pregnancies [1]. Although success with laparoscopic management is the same in single and multiple gestations, there have been very

\footnotetext{
M. Raja $(\bowtie) \cdot$ B. Donepudi $\cdot$ B. Whitlow

Essex Rivers Healthcare NHS Trust,

Department of Obstetrics and Gynaecology,

Turner Road,

Colchester CO4 5JL, UK

e-mail: malarraja@yahoo.com
}

few reported cases of twin tubal pregnancies managed laparoscopically.

\section{Case report}

A 37-year-old gravida 11 para $1^{+10}$ presented to Colchester General Hospital with a 2-day history of lower abdominal pain and mild PV bleeding. She had a trans-vaginal scan a week ago, which showed an empty uterus. Her last menstrual period was roughly 5 weeks ago, and she was not using any contraception. Her past history was significant for the fact that she had a left salpingostomy for a previous ectopic pregnancy and also had a right ovarian cystectomy. She never had an intrauterine contraceptive device or took ovulation-inducing drugs and she conceived naturally.

On examination she was in pain; her pulse rate was 103/ min and blood pressure (BP) 112/73. Abdominal examination revealed a soft and lax abdomen with no signs of peritonism. The patient declined a vaginal examination despite emphasising its importance. Clinically an ectopic pregnancy was suspected and her beta-human chorionic gonadotrophin (HCG) level was 8,567 IU/l. A trans-vaginal scan showed an empty uterus with an endometrial thickness of $13 \mathrm{~mm}$.

In the right adnexa a complex mass of approximately $8 \times 5 \times 4 \mathrm{~cm}$ was noted within which there were two distinct gestational sacs (Fig. 1). As she did not meet the criteria for methotrexate treatment (RCOG Green Top Guidelines) [2] an urgent laparoscopy was performed which confirmed the findings (Fig. 2) of twin tubal pregnancy on the right side. As there were dense adhesions on the left side, laparoscopic colorectal surgeons were involved in the adhesiolysis. She had a right salpingectomy using a harmonic scalpel and a bipolar scissors for fine dissection 


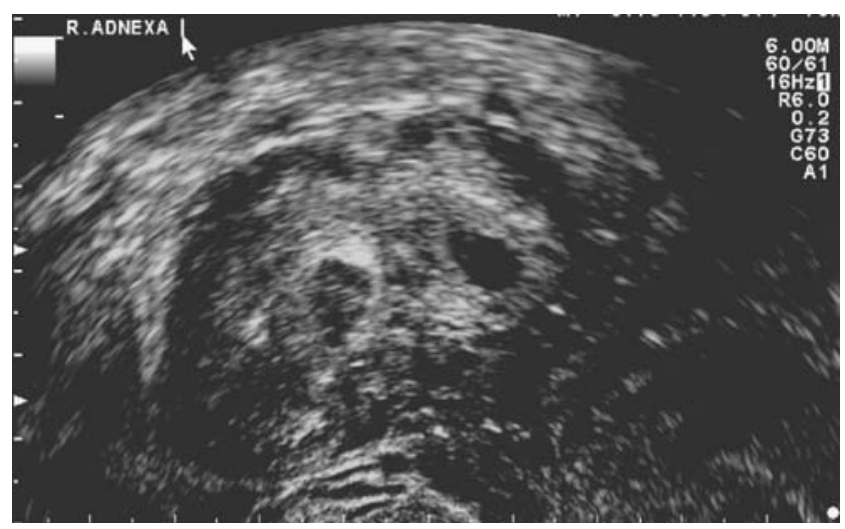

Fig. 1 Complex mass with two distinct gestational sacs in the right adnexa

and haemostasis. The post-operative period was uneventful and she was discharged on the second post-operative day.

\section{Discussion}

After DeOtt reported the first case of unilateral twin ectopic pregnancy in 1891 more than 100 cases have been reported to date. It has only recently been possible with the availability of high-resolution TV probes for these twin ectopic pregnancies to be diagnosed before tubal rupture. This case was notable for the fact that a correlation of the clinical findings, beta-HCG levels and the use of high-

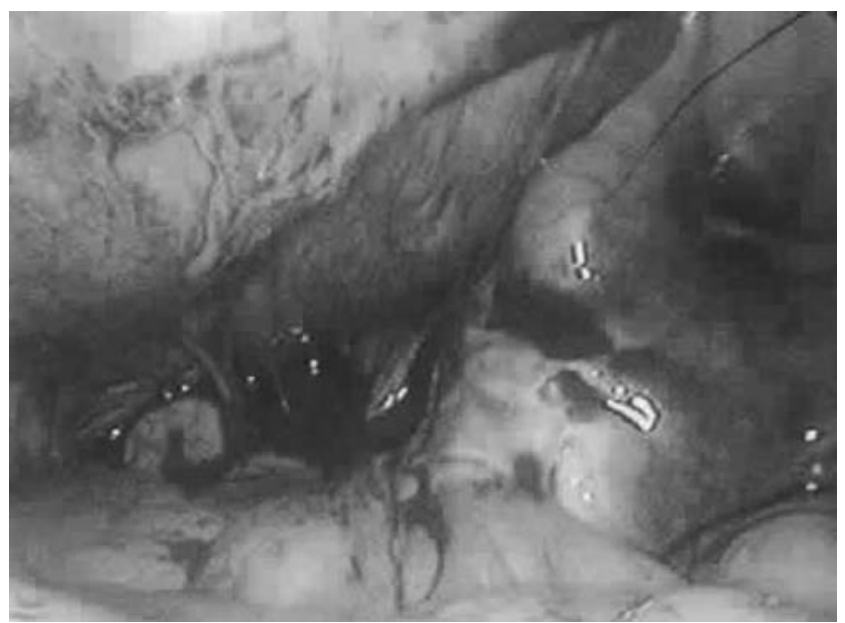

Fig. 2 Twin tubal pregnancy on the right side confirmed by laparoscopy resolution endovaginal probe helped clinch an early diagnosis, which was then managed with operative laparoscopy and salpingectomy. It should also be noted that it is not always possible to demonstrate two different sacs on the endovaginal ultrasound in very early cases (like the one we describe).

Bearing in mind the excess morbidity and mortality associated with ectopic pregnancy and the fact that all women of child bearing age can potentially have an ectopic pregnancy and also because it is challenging to diagnose ectopic pregnancy on a clinical basis alone, we have to utilise all the available resources especially in the high risk groups to confidently include or exclude the possibility of an ectopic pregnancy.

Serial monitoring of beta-HCG levels and the use of high-resolution trans-vaginal ultrasonography have a vital role in excluding or including intrauterine pregnancy, and in the odd difficult case the use of diagnostic laparoscopy comes in handy and is a reliable tool in the armamentarium of a clinician. Alarm bells should ring about the possibility of a multiple ectopic gestation (among the other rare differential diagnoses) when there is no demonstrable intrauterine gestational sac in the presence of abnormally rising HCG titres [3, 4].

It is notable that we did not have all the classic symptoms and signs of ectopic pregnancy but it is only the high index of suspicion in an high risk lady that led to a timely diagnosis and treatment and more importantly early discharge because of laparoscopic surgery. We cannot emphasise enough of what is said, "if you think of ectopic pregnancy, you are always sure to diagnose it" [5].

\section{References}

1. Hanchate V, Garg A, Seth R, Rao J, Jadav PJ, Karayil D (2002) Transvaginal sonographic diagnosis of live monochorionic twin ectopic pregnancy. J Clin Ultrasound 30(1):52-56

2. Royal College of Obstetricians and Gynaecologists (2004) The management of tubal pregnancy. Guideline no. 21. RCOG, London

3. Ash KM, Lyons EA, Levi CS, Lindsay DJ (1991) Endovaginal sonographic diagnosis of ectopic twin gestation. J Ultrasound Med 10(9):497-500

4. Eddib A, Olawaiye A, Withiam-Leitch M, Rodgers B, Yeh J (2006) Live twin tubal ectopic pregnancy. Int J Gynaecol Obstet 93:154155

5. Dorfman SF (1984) Ectopic pregnancy. "Thinking ectopic," key to diagnosis. Postgrad Med 76:65-68 